\title{
O EFEITO DA GEOMETRIA DO VAZAMENTO NA NUVEM DE GÁS INFLAMÁVEL: UMA ANÁLISE SOB A ÓTICA DA SEGURANÇA DO PROCESSO
}

\author{
C. M. de GODOY ${ }^{1}$, T. D. FERREIRA ${ }^{1}$ e S. S. V. VIANNA ${ }^{1}$ \\ ${ }^{1}$ Universidade Estadual de Campinas, Departamento de Engenharia Química \\ E-mail para contato: c116438@dac.unicamp.br
}

\begin{abstract}
The necessity of a new jet modelling approach in safety studies has been discussed. Differences between the jet behaviour originated by different orifice shapes have been observed. The current work presents an analysis about the differences between flammable gas clouds formed by axisymmetric jets (usually used in safety studies) and high aspect ratio jets (that has been suggested by some authors as the best way to represent real leak scenarios in industry, such as flange leakage). The RANS (Reynolds Averaged Navier Stokes) CFD modelling approach is used. An offshore module was considered and a gas leak was placed into the geometry. The work is also focused on the efficiency of the computational tool ANSYS - CFX 15.0 in reproducing the high aspect ratio jets (HARJ). The results indicated the need of great computational effort in order to represent the short distance behaviour of high aspect ratio jets. Besides, it had been observed that the gas clouds originated from HARJ are formed closer to the orifice than the clouds obtained by axisymmetric jets. For HARJ, the total flammable volume is less than or equal to the volume of the clouds originated by axisymmetric jets.
\end{abstract}

\section{INTRODUÇÃO}

A formação das nuvens inflamáveis é afetada diretamente pelos parâmetros que influenciam o grau de mistura do material inflamável com o ar, destacando-se os efeitos de curta distância (comportamento do vazamento) e os efeitos de longa distância (ventilação e condição atmosférica). Os efeitos de curta distância se tornam mais importantes conforme aumenta a velocidade de desprendimento, sendo muito influenciados pelo tipo de geometria do orifício que origina o vazamento (Cleaver et al., 1994; Lees, 2005).

Atualmente, a grande maioria dos estudos de análise de risco adota o modelo do jato axissimétrico para simulações de vazamentos. Esse modelo engloba os jatos que apresentam simetria em relação à sua linha central apresentando em sua estrutura duas regiões características: a região de núcleo potencial e região de decaimento axissimétrico (Figura 1-a). A utilização do jato axissimétrico está baseada principalmente no fato de que este tipo de jato tem um comportamento muito bem conhecido e fácil de ser modelado (Wakes et al., 2001).

Entretanto, o que se observa na prática é que a maioria dos vazamentos é ocasionada por falhas 
nas tubulações como rupturas, problemas relacionados à corrosão ou falhas nos flanges. Nesses casos, a geometria do orifício por onde ocorre o vazamento é diferente daquela prevista no modelo de jato axissimétrico (orifício circular). Assim, conforme proposto por Wakes et al. (2001), uma abordagem mais realística para a caracterização dos vazamentos, pelo menos numa escala próxima a sua região de origem, recai na modelagem de um jato retangular de elevada razão de aspecto (HARJ). Tais jatos têm comportamento tridimensional e apresentam três regiões distintas de decaimento de velocidade (Figura 1-b): o núcleo potencial, a região de decaimento característico e a região que se assemelha a de decaimento axissimétrico (Wakes et al., 2001).

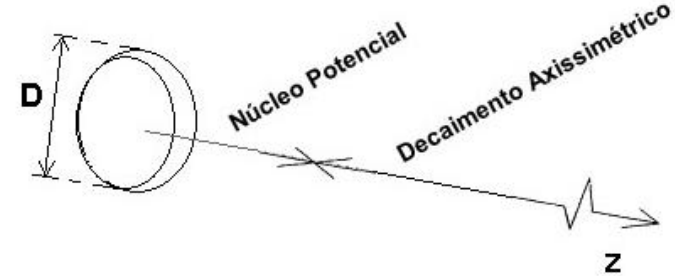

(a)

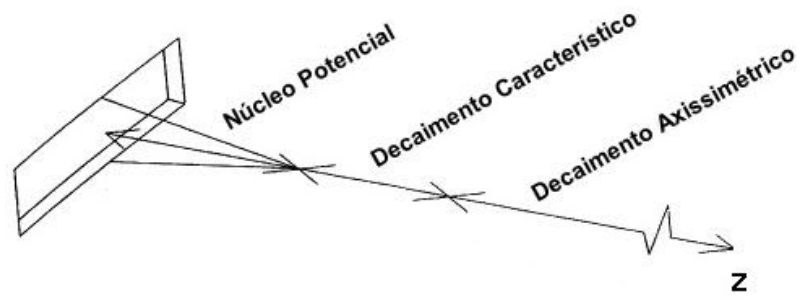

(b)

Figura 1. Esquema de jatos destacando as regiões de decaimento: (a) Jato axissimétrico; (b) Jato retangular de elevada razão de aspecto.

O presente estudo visa à investigação da influência da geometria do orifício de vazamento na nuvem de gás inflamável. Para tanto, fez-se uso da modelagem fluidodinâmica computacional $(C F D)$ com abordagem RANS (Reynolds-Averaged Navier-Stokes), e avaliou-se as diferenças entre as nuvens de gás inflamável formadas por jatos axissimétricos e jatos de elevada razão de aspecto.

\section{METODOLOGIA}

Este trabalho foi dividido em duas etapas principais: validação da ferramenta computacional ANSYS - CFX 15.0 para modelar jatos de elevada razão de aspecto e a simulação da dispersão de gás em uma plataforma de petróleo a partir de vazamentos originados por jatos axissimétricos e jatos de elevada razão de aspecto.

Para a comprovação da eficiência da modelagem computacional adotada, foram consideradas duas geometrias distintas de flanges com suas vedações rompidas, de forma a originar diferentes jatos retangulares de elevada razão de aspecto. As geometrias adotadas seguem a identificação proposta por Wakes et al. (2001), ressaltando o ângulo de rompimento da vedação junto ao duto de fluido $(\alpha)$, o ângulo de rompimento da vedação na superfície exterior do flange $(\beta)$ e a espessura da vedação do flange em milímetros $(\delta)$, sendo 00_90_02 e 70_45_02 (Figura 2-a). Os jatos foram definidos de forma a fixar a vazão mássica de ar em $0,15 \mathrm{~kg} / \mathrm{s}$ para a geometria 70_45_02 e 0,18 kg/s para a 00_90_02. Foi considerado um escoamento incompressível, isotérmico $\left(25^{\circ} \mathrm{C}\right)$ e em estado estacionário. Para estas simulações apenas a região de falha do flange foi reproduzida (como mostrado na Figura 2-b) e o domínio computacional foi definido como uma caixa de base retangular $(\mathrm{x}=0,3 \mathrm{~m}$ e $\mathrm{z}=2 \mathrm{~m})$ e 2 metros de altura. 


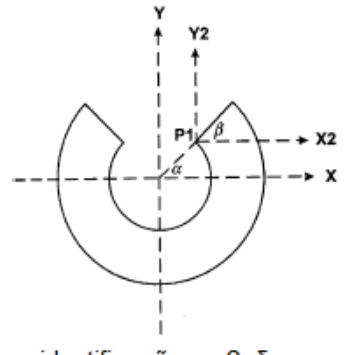

identificação: $\alpha \_\beta \_\delta$

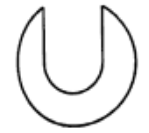

00_90_02

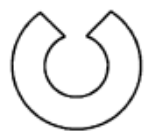

70_45_02

(a)

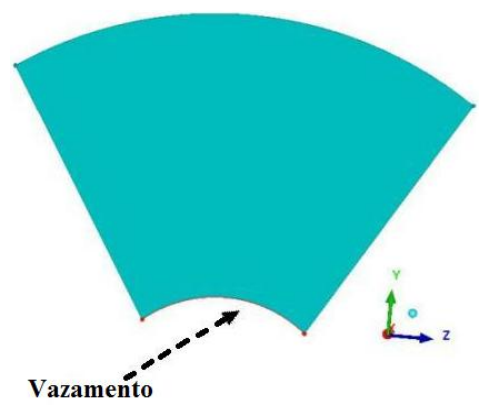

(b)

Figura 2 - (a) Esquema da identificação do vazamento destacando os ângulos característicos $\alpha$ e $\beta$ e as duas geometrias adotadas Adaptado (Wakes et al., 2001). (b) Representação da região de falha do flange para fins de modelagem e simulação no CFX 15.0.

$\mathrm{Na}$ etapa de análise da dispersão de gás compararam-se os tamanhos das nuvens inflamáveis originadas por jatos de elevada razão de aspecto e jatos axissimétricos. Para isso, foi considerada como geometria uma plataforma de petróleo típica (Figura 3-a) onde foi introduzido um vazamento com diferentes formatos (flanges e orifícios circulares). Os vazamentos foram posicionados em cinco direções: $+\mathrm{x},-\mathrm{x},+\mathrm{y},-\mathrm{y}$ e $-\mathrm{z}$, de acordo com a orientação da plataforma. Além disso, foram consideradas oito direções de vento com uma velocidade de 6,5 m/s, conforme Figura 3-b.

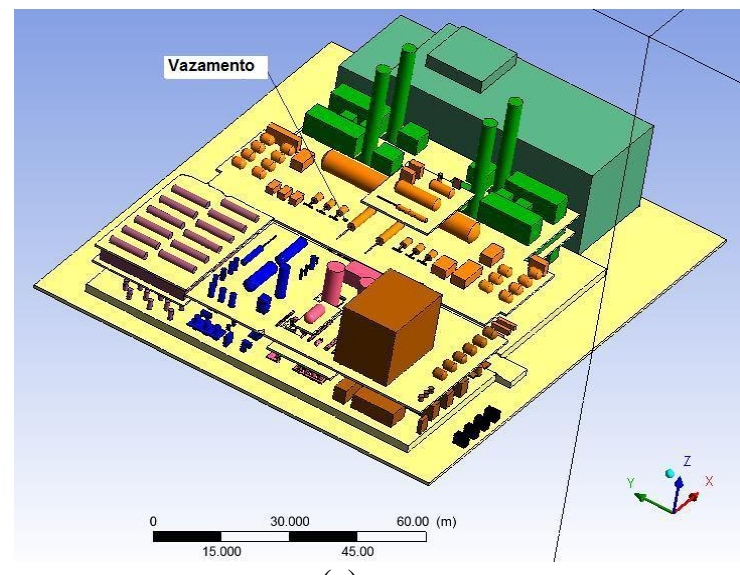

(a)

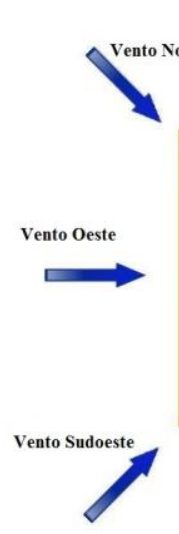

Figura 3 - Geometria da plataforma considerada destacando: (a) o posicionamento do vazamento e (b) as direções de vento consideradas (Ferreira, 2014).

A geometria dos flanges foi adaptada de forma a propiciar vazamentos capazes de originar nuvens inflamáveis de dimensões significativas (acima de $10 \mathrm{~kg} / \mathrm{s}$ ). Assim, foi utilizada a geometria 00_90_70 com razão de aspecto $=20$. Foi considerada uma linha de gás pressurizada com uma pressão média de $200 \mathrm{kgf} / \mathrm{cm}^{2}$ e a vazão mássica de gás natural no vazamento foi fixada em $30 \mathrm{~kg} / \mathrm{s}$. Nestas simulações a modelagem fenomenológica do vazamento envolveu um escoamento incompressível, com troca térmica e em estado estacionário.

Modelagem matemática: Em ambas as etapas, as equações resolvidas foram: conservação da massa, conservação do momento e o modelo de turbulência. Considerando as hipóteses de regime 
permanente e escoamento incompressível, as equações de conservação de massa e de momento resolvidas foram, respectivamente:

$$
\begin{aligned}
& \boldsymbol{\nabla} \cdot \boldsymbol{u}=0 \\
& \boldsymbol{u} \cdot \boldsymbol{\nabla}(\boldsymbol{u})=-\boldsymbol{\nabla} / \rho+v \boldsymbol{\nabla} \boldsymbol{u}^{2}+\boldsymbol{g}
\end{aligned}
$$

Foi utilisado o modelo de turbulência $k-\varepsilon$ no qual, para a obtenção dos valores dos parâmetros k (energia cinética turbulenta) e $\varepsilon$ (taxa de dissipação turbilhonar) aplicam-se as equações:

$$
\begin{aligned}
& \partial(\rho k) / \partial t+\boldsymbol{\nabla} \boldsymbol{u} \boldsymbol{u}=\boldsymbol{\nabla}\left[\left(\mu+\mu_{t} / \sigma_{k}\right) \boldsymbol{\nabla}\right]+P^{k b}+\left(P^{k}-\rho \varepsilon\right) \\
& \partial(\rho \varepsilon) / \partial t+\boldsymbol{\nabla} \boldsymbol{u} \varepsilon=\nabla\left[\left(\mu+\mu_{t} / \sigma_{\varepsilon}\right) \boldsymbol{\nabla} \varepsilon\right]+(\varepsilon / k)\left(C_{1 \varepsilon} P^{k}-C_{2 \varepsilon} \rho \varepsilon+C_{1 \varepsilon} P^{\varepsilon b}\right)
\end{aligned}
$$

Em que $\mathrm{C}_{1 \varepsilon}=1,44 ; \mathrm{C}_{2 \varepsilon}=1,92 ; \sigma_{\mathrm{k}}=1,3$ e $\sigma_{\varepsilon}=1$. $\mu_{\mathrm{t}}$ é a viscosidade turbilhonar, dada segundo a relação: $\mu_{t}=C_{\mu} \rho k^{2} / \varepsilon \quad\left(\operatorname{com} C_{\mu}=0,09\right)$.

$\mathrm{Na}$ etapa de análise da dispersão do gás, além dessas equações, a modelagem matemática contou também com a equação da energia que, para o escoamento em questão, pode ser expressa por:

$$
\rho \boldsymbol{\nabla} \cdot\left(\boldsymbol{u} \hat{C}_{p} T\right)=\boldsymbol{\nabla} \cdot\left(k_{T} \boldsymbol{\nabla} T\right)+\mu \varphi_{v}
$$

\section{RESULTADOS}

Para avaliar a eficiência da ferramenta computacional ANSYS - CFX 15.0 na modelagem de jatos de alta razão de aspecto foi analisado o comportamento da velocidade na linha central do jato. A Figura 4 mostra a comparação entre os resultados das simulações e os dados experimentais fornecidos por Wakes et al. (2001) utilizando malhas com diferentes refinamentos na região do vazamento do flange e no restante do domínio computacional. Na região do flange, os tamanhos dos elementos da malha variaram entre 0,5 e 1 milímetro enquanto no restante do domínio computacional o tamanho dos elementos varia entre 20 e 50 milímetros. A sigla "l" na identificação da geometria indica a malha mais grosseira, a sigla " $v$ " representa uma malha com elementos de tamanho intermediário e a sigla " $d s$ " representa uma malha com um refinamento especial ao longo da linha central do jato. É possível observar que, para as duas geometrias analisadas a malha " $d s$ " forneceu os melhores resultados e, por isso, esta malha foi adotada nas análises subsequentes.

A Figura 5 fornece os resultados das simulações obtidos quando diferentes esquemas de solução numérica são adotados. O termo "High Resolution" se refere a um esquema híbrido de solução assim denominado pelo CFX, em que ora o cálculo é feito usando o método upwind e ora usando um esquema de ordem superior, dependendo do gradiente de velocidade. No caso da sigla $\mathrm{SBF}=1$, todos os cálculos são realizados usando um esquema de segunda ordem. Comparando os resultados das simulações com os dados experimentais, é possível verificar que, para ambas as geometrias consideradas o método de solução SBF fornece os melhores resultados. 


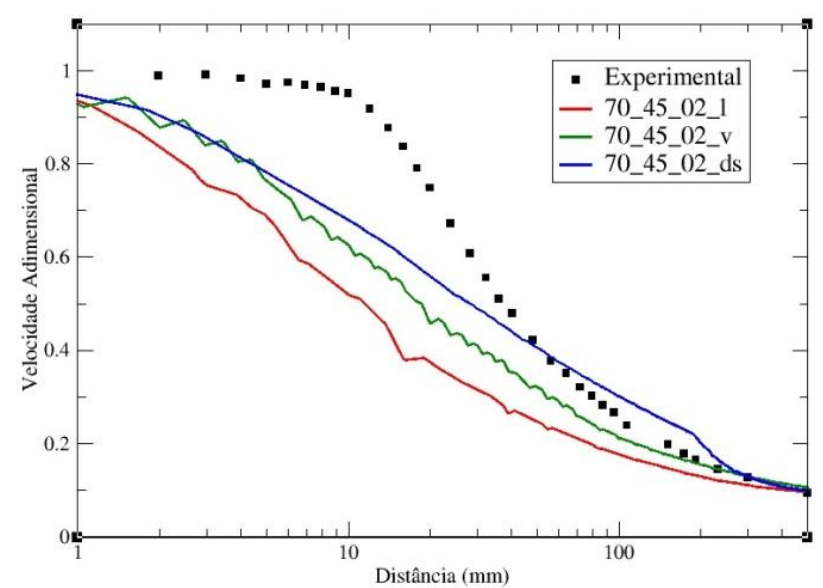

(a)

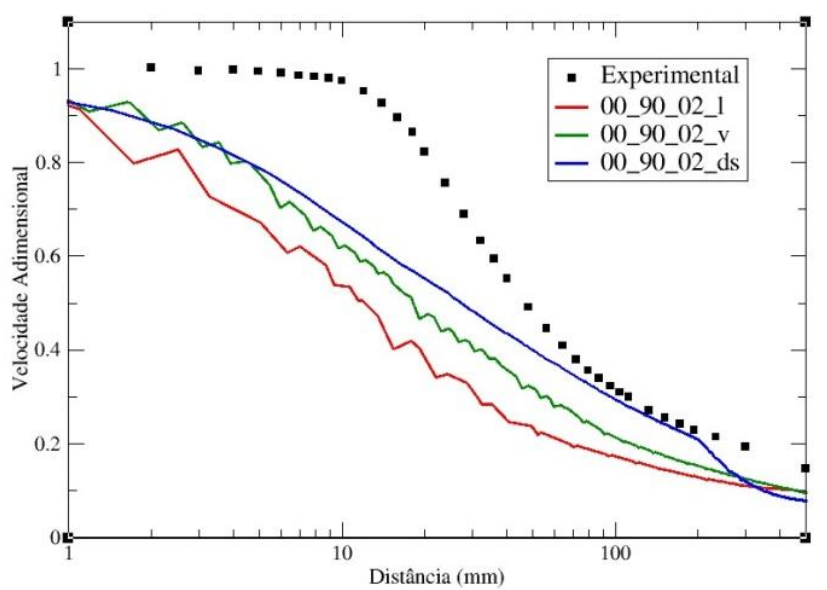

(b)

Figura 4 - Decaimento da velocidade na linha central do jato considerando diferentes malhas para as geometrias (a) 70_45 e (b) 00_90.

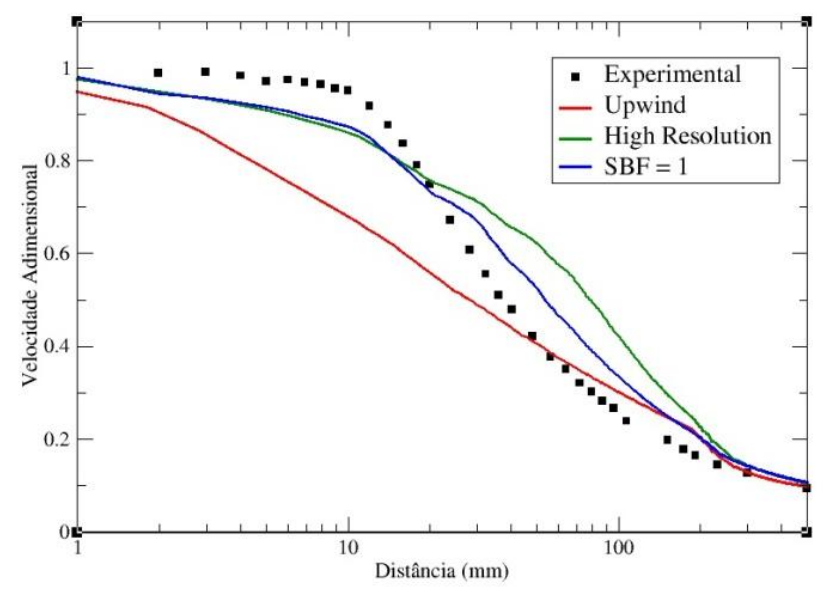

(a)

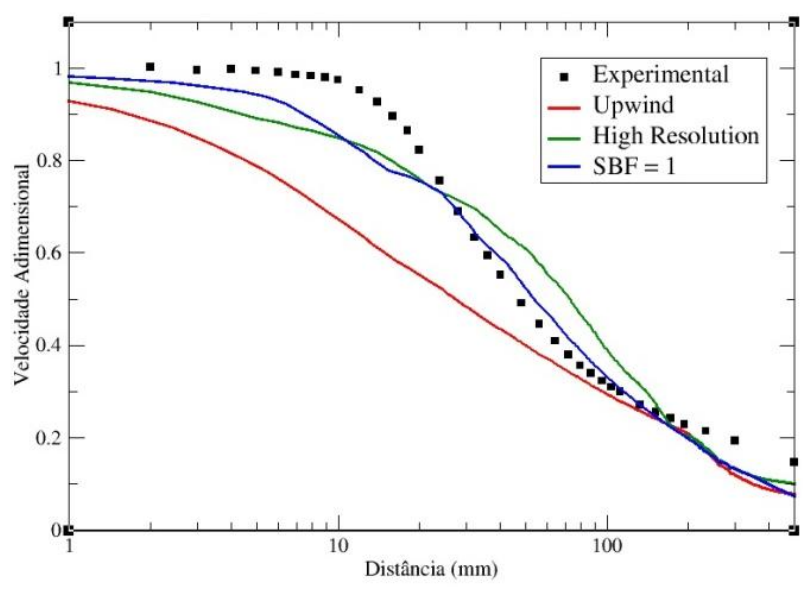

(b)

Figura 5 - Decaimento da velocidade na linha central do jato considerando diferentes esquemas de solução numérica nas simulações do vazamento em flanges com as geometrias (a) 70_45 e (b) 00_90.

$\mathrm{Na}$ análise de dispersão de gás na plataforma, foram analisados os tamanhos de nuvens inflamáveis formadas a partir de jatos axissimétricos e de elevada razão de aspecto e a distância a partir da origem do jato em que a nuvem de gás inflamável começa a ser formada. A Figura 6 mostra a comparação entre os tamanhos de nuvem de gás inflamáveis formadas a partir de jatos axissimétricos e HARJ considerando vazamentos nas direções x, y e z. É possível verificar que, para a grande maioria dos casos, os jatos de elevada razão de aspecto originam nuvens inflamáveis menores do que os jatos axissimétricos. Tal observação pode estar relacionada ao fato de que quando são usados jatos de elevada razão de aspecto há uma intensa mistura entre o ar e o gás vazado, de forma que a mistura ar-gás final esteja, em sua maior parte, abaixo do limite inferior de inflamabilidade. Este resultado é diferente da análise proposta por Wakes et al. (2001) que sugere que jatos de elevada razão de razão de aspecto originem maiores nuvens inflamáveis do que jatos axissimétricos. 


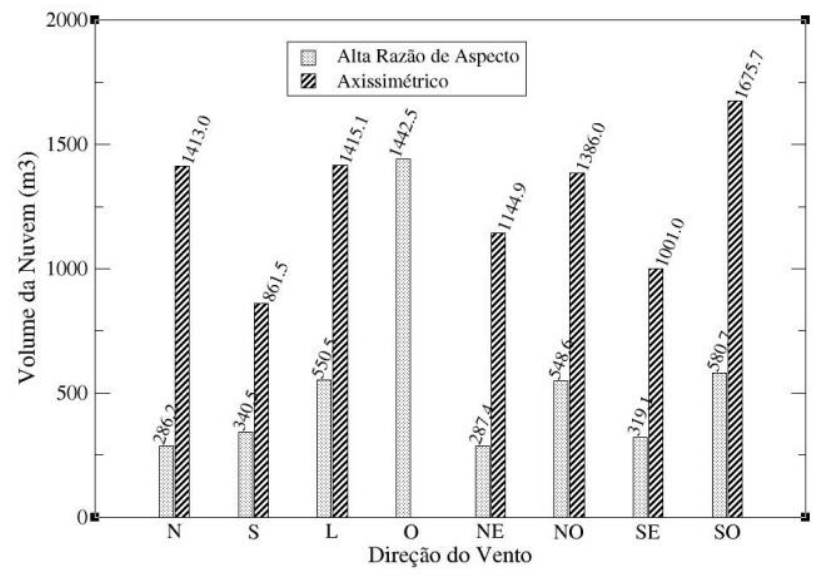

(a)

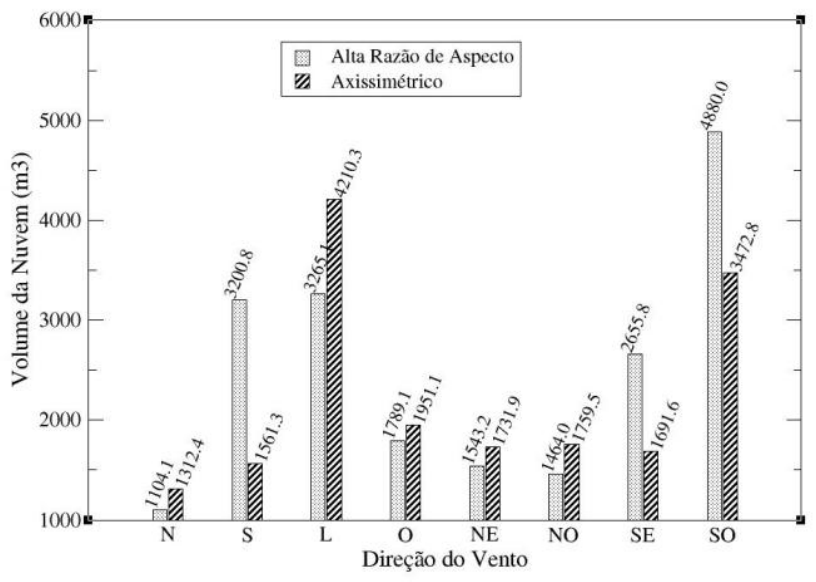

(c)

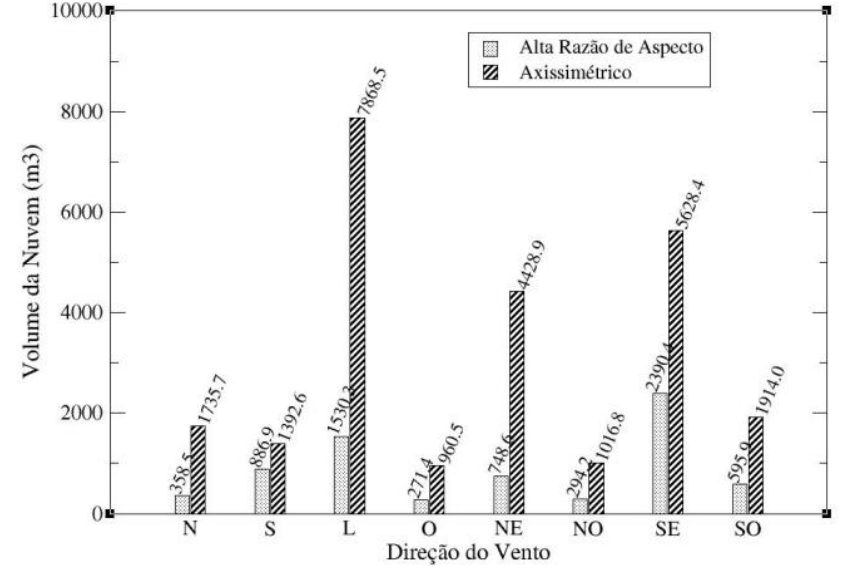

(b)

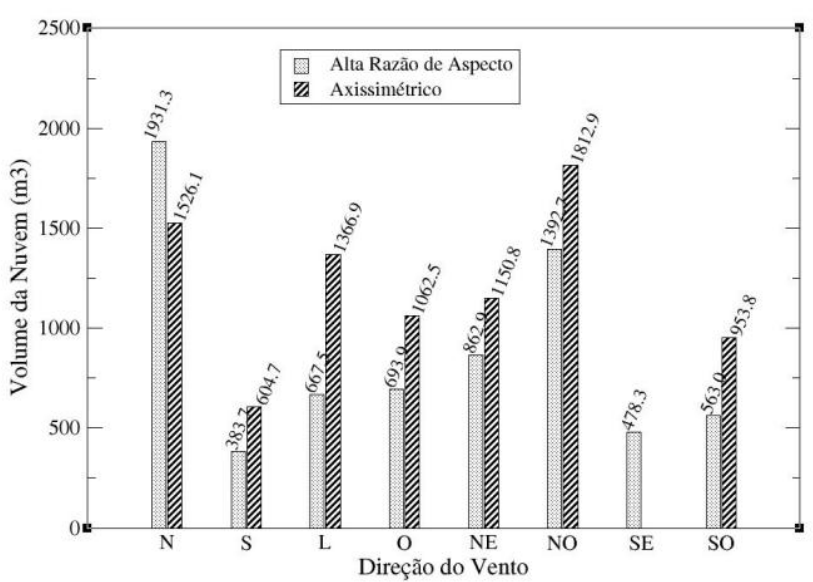

(d)

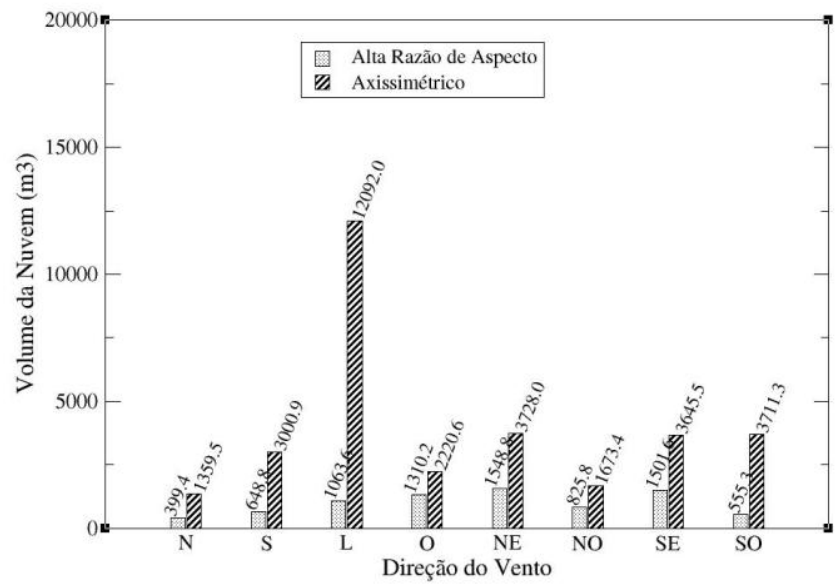

(e)

Figura 6 - Comparação entre os tamanhos de nuvem de gás inflamável originadas a partir de jatos axissimétricos e jatos de elevada razão de aspecto para as oito possíveis direções de vento e considerando vazamentos na direção (a) - x, (b) $+\mathrm{x},(\mathrm{c})-\mathrm{y},(\mathrm{d})+\mathrm{y}$ e (e) $-\mathrm{z}$. 
Para avaliar a distância a partir da origem do jato em que a nuvem de gás inflamável começa a ser formada, a região inflamável foi identificada num plano que corta o eixo central do jato. A Figura 7 mostra um exemplo de dispersão em que o vazamento está na direção $+x$ e o vento tem origem no leste. A mesma análise foi feita usando dez cenários com diferentes configurações de direção de vento e vazamento (Figura 8) e é possível verificar em todos eles que as nuvens formadas a partir de jatos de elevada razão de aspecto se tornam inflamáveis mais próximo à origem do jato que as formadas a partir de jatos axissimétricos. Este resultado é semelhante à análise feita por Wakes et al. (2001).

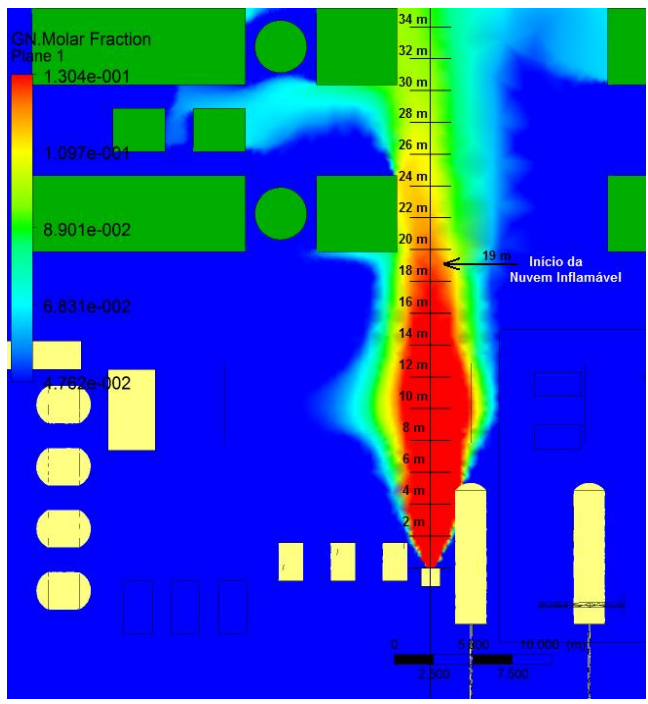

(a)

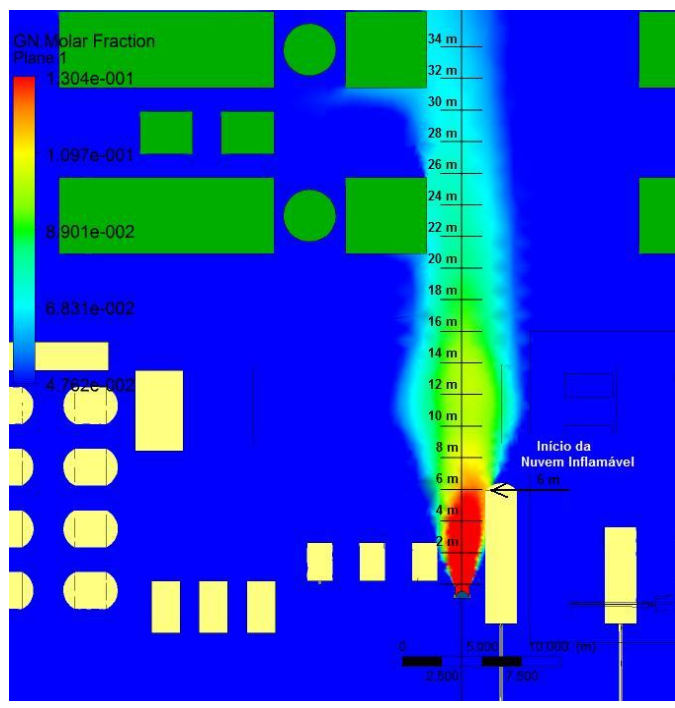

(b)

Figura 7 - Região inflamável da nuvem de gás em um plano no eixo central do jato considerando a nuvem sendo formada a partir de (a) um jato axissimétrico e (b) um jato de elevada razão de aspecto. Em vermelho está a região da nuvem em que a concentração do gás está acima do limite superior de inflamabilidade (para o metano, 15\% V/V) enquanto em azul está a região da nuvem em que a concentração do gás está abaixo do limite inferior de inflamabilidade (para o metano, $5 \% \mathrm{~V} / \mathrm{V}$ ).

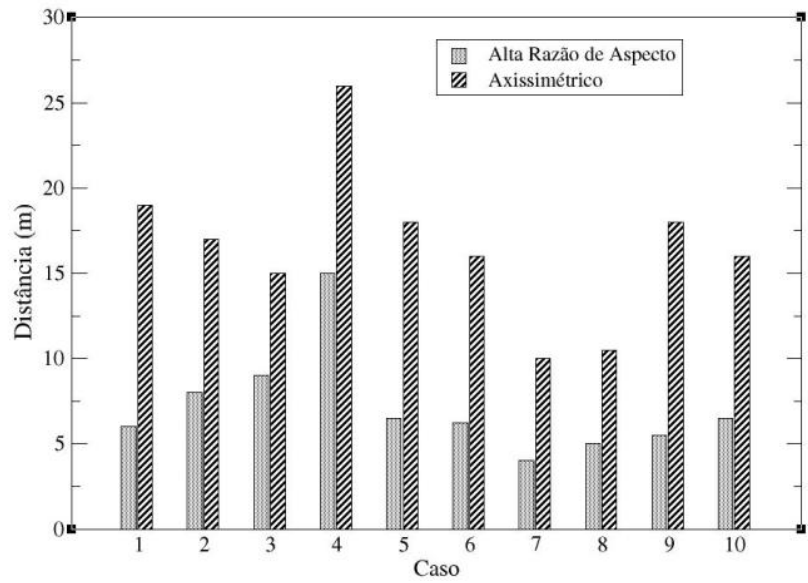

Figura 8-Comparação entre as distâncias a partir da origem do jato em que a nuvem de gás inflamável começa a ser formada para jatos axissimétricos e jatos de elevada razão de aspecto em dez diferentes casos considerando cinco direções de vento (N, S, L, O, NO) e vazamentos nas direções $+\mathrm{x},-\mathrm{x},+\mathrm{y}$ e $-\mathrm{y}$. 


\section{CONCLUSÕES}

Foi possível avaliar a influência da geometria do orifício de vazamento do jato na nuvem de gás inflamável.

Primeiramente, dois casos experimentais de vazamentos a partir de jatos de elevada razão de aspecto foram reproduzidos usando o software CFX-15.0. Em ambos os casos a melhor concordância entre os resultados das simulações e os dados experimentais foi obtida usando uma malha extremamente refinada e aplicando um esquema numérico de alta ordem para solução das equações, o que eleva consideravelmente o esforço e custo computacional para realizar este tipo de simulação.

Em relação à influência da geometria do orifício do vazamento no tamanho da nuvem de gás inflamável, observou-se que jatos de elevada razão de aspecto formam nuvens inflamáveis menores que os jatos axissimétricos. Pode-se considerar, então, que em uma abordagem mais conservadora para modelagem de jatos em análise de risco deve-se considerar jatos axissimétricos (com orifício de origem circular).

Outra importante análise é que as nuvens de gás inflamável originadas a partir de jatos de elevada razão de aspecto são formadas mais próximas ao vazamento quando comparadas às nuvens originadas por jatos axissimétricos.

\section{REFERÊNCIAS}

CLEAVER, R.P.; HUMPHREYS, C. E.; ROBINSON C. G. Accidental Generation of Gas Clouds on Offshore Process Installations. J. Loss Prev. Process Ind., v. 7, n. 4, p. 273-280, 1994.

FERREIRA, T. D. Developing a Mathematical Model for Prediction of Flammable Gas Cloud Size Based on CFD and Response Surface Methodology. Dissertação (Mestrado em Engenharia Química), Falculdade de Engenharia Química, Universidade Estadual de Campinas. Campinas, 2014.

LEES, F. P. Loss Prevention in the Process Industries - Hazard Identification, Assessment and Control. 3 ed. Oxford: Butterworth-Heinemann, 2005.

WAKES, S. J.; HOLD $\varnothing$, A. E.; MEARES, A. J. Experimental investigation of the effect orifice shape and fluid pressure has on high aspect ratio cross-sectional jet behavior. Journal of Hazardous Materials, A.89, p. 1-27, 2001. 